\title{
THEMEN
}

Prof. Dr. H. Schneider/Wissenschaftliche Mitarbeiterin J. Busch

\section{Der Lebensretter als Mörder? \\ Der „Organspendeskandal“ an den Grenzen der Strafrechtsdogmatik}

\section{A. Der Organspendeskandal}

Der „Göttinger Organspendeskandal“ ist seit seiner Aufdeckung im Sommer 2012 in der Presse als größter deutscher Medizinskandal bekannt geworden. Auch in anderen Universitätskliniken soll es zu vergleichbaren Unregelmäßigkeiten in der Listung der Transplantationspatienten gekommen sein. Die Strafverfahren gegen die beteiligten Transplantationsmediziner sind in unterschiedlichen Stadien, keines ist rechtskräftig abgeschlossen. Nur in Göttingen verhandelt bereits das Schwurgericht in einem aufsehenerregenden Prozess wegen des Verdachts des versuchten Totschlags in elf Fällen.

Der Medienberichterstattung ${ }^{1}$ sowie den mittlerweile publizierten Beschlüssen des Landgerichts ${ }^{2}$ und Oberlandesgerichts Braunschweig ${ }^{3}$ ist folgender Sachverhalt zu entnehmen: Am Göttinger Universitätsklinikum habe der angeklagte Transplantationsmediziner Aiman O. den Gesundheitszustand mehrerer sich bei ihm in Behandlung befindender Patienten unrichtig erhoben oder vorsätzlich falsch dokumentiert, um eine zügige und vorrangige Aufnahme auf die Warteliste und folglich eine schnelle Zuteilung eines Spenderorgans für seine Patienten zu erreichen. Diese Daten habe er sodann an die zentrale Spenderorganvermittlungsstelle Eurotransplant weitergegeben. Wartet ein Patient auf die Transplantation einer Spenderleber, erfolgt die Organzuteilung auf Grundlage des Model End Stage Liver Disease (MELD) Scores. Dieses Instrument dient der Abschätzung des Mortalitätsrisikos auf der Basis verschiedener Laborparameter und spiegelt so die Dringlichkeit einer Lebertransplantation wider. Ausweislich der Anklage sei

$1 \mathrm{Http} / /$ www.faz.net/aktuell/gesellschaft/prozessauftakt-im-transplantationsskandal-das-warmeine-liebe-zum-leben-12537899.html; http://www.zeit.de/wissen/gesundheit/2013-08/organspende-prozess-goettingen, http://www.taz.de/!122022/, abgerufen am 25.09.2013; FAZ v. 02.09.2013, 9 „Experiment mit 14 Toten“.

2 LG Braunschweig, Beschluss vom 11.02.2013 - 9 Qs 20/13.

3 OLG Braunschweig, Beschluss vom 20.03.2013 - Ws 49/13, Entscheidung über die weitere Beschwerde gem. $₫ 310$ Abs. 1 Nr. 1 StPO gegen die Anordnung von Untersuchungshaft im Göttinger Verfahren. 
der bevorzugte Patient aufgrund der Manipulation seines Arztes auf der Warteliste für ein Spenderorgan so weit nach oben gerückt, dass ihm alsbald eine Spenderleber zugeteilt werden konnte. In der darauffolgenden Zeit sei es zum Tod anderer Patienten gekommen, deren Namen sich auf der Warteliste befunden haben. Diese hätten entweder kein Spenderorgan bekommen oder die Transplantation sei mit zeitlicher Verzögerung erfolgt.

Seit der Häufung derartiger Schlagzeilen verstärkt sich die Skepsis der Bevölkerung gegenüber dem Gesundheitssystem. Die Zahl der Organspender in Deutschland sank nach Angaben der Deutschen Stiftung für Organtransplantation im ersten Halbjahr 2013 um 20 Prozent $^{4}$. Verunsicherung herrscht auch in Rechtswissenschaft und Medizin vor. Diskutiert wird die „Neuorganisation des Transplantationswesens in Deutschland“5. Strafrechtsdogmatisch ${ }^{6}$ geht es um grundlegende Probleme der Dogmatik des Allgemeinen Teils, um Fragen der Kausalität, der Abgrenzung zwischen Vollendung und Versuch, den Bezugspunkten des Vorsatzes und im Ergebnis um die Verurteilung wegen eines Kapitaldeliktes, möglicherweise sogar wegen Mordes ${ }^{7}$, oder Freispruch.

\section{B. Rechtsfragen im Zusammenhang mit der Anwendbarkeit der Tötungsdelikte}

\section{Vollendeter Totschlag}

\section{Corpus Delicti}

Schon das corpus delicti, der Ausgangspunkt für den kausalen Regress ad personam, ist in den Fallkonstellationen des Transplantationsskandals nicht ohne weiteres auszumachen. Ausgangspunkt der Suche ist die zentrale Warteliste für Transplantationen ${ }^{8}$ im Zeitpunkt der Datenmanipulation als Tathandlung im Sinne des $\$ 212$ StGB. Nur wer zu diesem Zeitpunkt oberhalb der eigentlichen Platzierung des zu Unrecht bevorzugten

4 http://www.heute.de/Organspende-Skandal-Prozess-startet-29335858.html, abgerufen am 04.09.2013.

5 Protokoll des 116. Deutschen Ärztetages in Hannover, TOP I.

6 Die bisher (Stand der Bearbeitung 30.09.2013) einzigen Veröffentlichungen zu den genannten Problemen des Transplantationsskandals sind die Arbeiten von Kudlich NJW 2013, 217ff. und Schroth NStZ 2013, 437ff.

7 Je nach Fallgestaltung ist es denkbar, dass für die Manipulationen finanzielle Motive ausschlaggebend sind (hohe Erlöse bei durchgeführter Transplantation, Steigerung des leistungsbezogenen Vergütungsanteils durch Erreichen bestimmter Scorecard-Vorgaben), es um die Steigerung der Reputation als Transplantationsmediziner bzw. des Transplantationszentrums oder um altruistische Beweggründe (schnelle Hilfe für die eigenen Patienten, Aufbegehren gegen die als verfassungswidrig empfundenen Kriterien der Organvergabe, vgl. OLG Braunschweig, Beschl. vom 20.03.2013 - Ws 49/13) geht. In den beiden zuerst genannten Gründen ist die Annahme von Habgier bzw. niedrigen Beweggründen vorbehaltlich der gebotenen restriktiven Auslegung der Merkmale des $\mathbb{2} 211$ StGB (zur Lösung des BGH, vgl. umfassend, MK-Schneider, $\$ 211$, Rn. 39ff.) jedenfalls nicht ausgeschlossen. Liegt ein „Motivbündel“ vor, wäre der beherrschende Tatantrieb zu ermitteln, BGH, Urteil vom 06.10.2004 - 1 StR 286/04, NStZ 2005, 332-334.

8 \12 III TPG.

NK 25. Jg. 4/2013 
Patienten für ein Spenderorgan gelistet ist und bis zur Anklageerhebung verstirbt, kommt als Opfer eines vollendeten Totschlags gem. $\$ 212$ StGB in Betracht ${ }^{9}$. Alle anderen Patienten, deren Namen für ein Spenderorgan vor dem bevorzugten Patienten auf der Warteliste stehen, könnten allerdings zumindest Opfer eines versuchten Tötungsdelikts geworden sein.

Opfer ist in der einfachsten denkbaren Konstellation der durch Manipulation unmittelbar von seinem Listenplatz verdrängte Patient, soweit dieser keine Leber erhält und verstirbt. Eine Manipulation kann aber auch für mehrere Erfolge im Sinne des $\$ 212 \mathrm{StGB}$ verantwortlich sein, wenn man z.B. ${ }^{10}$ auf den Aspekt der manipulationsbedingten zeitverzögerten Organtransplantation abstellt. So liegt es ggf. in dem im Göttinger Verfahren ermittelten Fall, in dem ein Patient durch die Manipulation von Platz 34 auf Rang 2 „befördert" und mit einem Spenderorgan versorgt worden sei ${ }^{11}$. Auch die Patienten auf den Rängen 1 - $10^{12}$ hätten schließlich eine Leber erhalten, allerdings seien einige dieser transplantierten Patienten kurz nach der Operation verstorben. Für die strafrechtliche Prüfung kommen auch die Patienten auf den Rängen 3 - 10 als Opfer eines Tötungsdeliktes in Betracht, weil bei ihnen möglicherweise die Transplantation durch die Datenmanipulation verzögert wurde. Konstruiert man den dargestellten Fall insofern weiter, dass der Patient mit dem Listenplatz 11 stirbt, bevor er ein Organ erhält, kann der Tod ebenfalls mit der Manipulation in Verbindung gebracht werden, da der Person mit der Platznummer 10 ein Organ transplantiert wurde. Ob und inwieweit der angeklagte Mediziner im Göttinger Verfahren im Ergebnis allerdings für den Tod dieser Menschen zur Verantwortung gezogen werden kann, ist ein Problem der Erfolgszurechnung, nicht des tatbestandlichen Erfolges (siehe sogleich).

\section{Tatbestandsmäßiges Verhalten}

Aufgrund der Konzeption des $₫ 212$ StGB als reines Erfolgsdelikt kann grundsätzlich jedes Verhalten als tatbestandsmäßige Tötungshandlung in Betracht kommen ${ }^{13}$. Nach dem bisherigen Kenntnisstand stehen unterschiedliche Vorgehensweisen der Manipulation von Patientendaten im Raum. Zum Teil sei unzutreffend eine Dialysepflichtigkeit des Patienten angegeben worden. In anderen Fällen sei verschwiegen worden, dass die nach den Richtlinien der Bundesärztekammer vorgeschriebene Alkoholabstinenz von sechs Monaten nicht eingehalten wurde. Außerdem soll es zu einer Dokumentierung

9 Schmidt-Aßmann in Maunz/Dürig 2013, Art. 103 III GG, Rn. 285.

10 Denkbar wäre auch, dass der durch die Manipulation verdrängte Patient ein Organ ungleicher Qualität erhalten hat, wobei dieses einerseits „besser“, andererseits aber auch „schlechter“ sein kann.

11 FAZ v. 02.09.2013, 9.

12 FAZ v. 02.09.2013, 9: „Die Kammer forschte nach, was aus den übrigen Patienten auf den Rängen eins bis zehn wurde“.

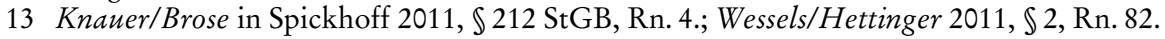


falscher Blutwerte gekommen bzw. soll eine Behandlung zur Verbesserung der Blutwerte absichtlich unterlassen worden $\operatorname{sein}^{14}$.

Insofern besteht das tatbestandsmäßige Verhalten auch dann in einem aktiven Tun ${ }^{15}$, wenn man - was strafrechtsdogmatisch weder weiterführend noch erforderlich ist - die Manipulation der Daten als Abbruch eines bereits von dritter Seite eingeleiteten rettenden Kausalverlaufs für den verstorbenen Patienten ansieht. Denn diese Sachverhalte, die in der Regel reine Lehrbuchkonstruktionen darstellen (z.B. das Zurückhalten eines zu einem Lawinenunglück gesandten Rettungshundes ${ }^{16}$ ), werden nach ganz herrschender Meinung ${ }^{17} \mathrm{zu}$ Recht ebenfalls als Fälle des aktiven Tuns qualifiziert ${ }^{18}$.

Dass die Tötungshandlung gleichzeitig zur Rettung des Lebens eines anderen Menschen führt, stellt ein denkbares ethisches Dilemma des Arztes dar, der je nach Sachlage gegebenenfalls „eigene“ Patienten bestmöglich mit dem knappen Gut der Spenderorgane versorgen möchte, dogmatisch erheblich ist dieser Umstand freilich nicht ${ }^{19}$.

\section{Kausalität}

Schwierigkeiten ergeben sich bei den vorliegenden Fallgestaltungen insbesondere bei der Feststellung der Kausalität. Nach der herrschenden Äquivalenztheorie ${ }^{20}$ ist durch Anwendung des „hypothetischen Eliminationsverfahrens“21 zu ermitteln, was geschehen wäre, wenn der Täter die Manipulationen an den Patientendaten nicht vorgenommen hätte. Eliminiert man gedanklich die Weitergabe der falschen Patientendaten an die zuständige Vermittlungsstelle für Organspenden in den Benelux-Ländern, Deutschland, Österreich, Slowenien, Kroatien und Ungarn „Eurotransplant“, hätte die bevorzugte Person nicht den entsprechenden Listenplatz erhalten und die unter korrekten Vergabekriterien an diesem Platz stehende und nun unmittelbar nachfolgende Person wäre zu diesem Zeitpunkt mit dem Organ versorgt worden. Allerdings ist dies die einzige (halbwegs $^{22}$ ) sichere Feststellung, die mit Hilfe der Anwendung der Äquivalenztheorie und

$14 \mathrm{http} / /$ www.faz.net/aktuell/gesellschaft/kriminalitaet/transplantationsskandal-wir-verlierenpatienten-12557026.html, abgerufen am 04.09.2013. Insofern steht auch eine vorsätzliche Körperverletzung zum Nachteil des unterversorgten Patienten im Raum.

15 Näher Schroth NStZ 2013, 437, 443: „Die durch Manipulation herbeigeführte Nichtzuteilung eines Organs durch Eurotransplant ist ein Unterlassen, das durch ein aktives Tun - Manipulation der Krankenakten - veranlasst wurde.".

16 Vgl. die bereits von Kaufmann 1959, 195ff. entwickelten Beispiele.

17 Wessels/Beulke 2012, Rn. 701.

18 Kindhäuser 2011, \$35, Rn. 3.

19 Auch $\$ 34$ StGB ist nicht einschlägig, vgl. insoweit zutreffend OLG Braunschweig, Beschluss vom 20.03.2013 - Ws 49/13 (juris Rn. 53). Strafzumessungsrechtlich sind die Motive des Täters demgegenüber von Bedeutung.

20 Lenckner/Eisele in Schönke/Schröder 2010, Vorbem. zu $\$ 13$ ff., Rn. 73; Kühl in Lackner/Kühl 2011, Vorbem. zu \$13, Rn. 9.

21 LK-Jescheck, Vorbem. zu \13, Rn. 50; Lenckner/Eisele in Schönke/Schröder 2010, Vorbem. $\mathrm{zu} \ 13$ ff., Rn. 73.

22 Die Vergabekriterien lassen nicht immer mit mathematischer Exaktheit die Bestimmung eines konkreten alternativen Empfängers zu, näher Schroth NStZ 2013, $437 \mathrm{ff}$.

NK 25. Jg. 4/2013 
des Eliminationsverfahrens getroffen werden kann. Denn die Transplantation kann, muss aber nicht zu einer Lebensrettung oder zumindest, was unter Kausalitätsaspekten nach Auffassung der Rechtsprechung ausreichend wäre, zu einer Lebensverlängerung „mindestens im Bereich von Stunden“23 führen ${ }^{24}$. Sie bewirkt im Gegenteil sogar eine Lebensverkürzung, wenn der Patient bei oder kurz nach ${ }^{25}$ der Operation verstirbt, ohne diese aber noch geraume Zeit weitergelebt ${ }^{26}$ haben würde. Aufgrund des morbiden Gesamtstatus des Patienten lässt die Bedingungstheorie insofern keine sichere Kausalitätsfeststellung zu. Ein Ausweichen auf eine „kausalitätsersetzende Risikoerhöhungsleh-

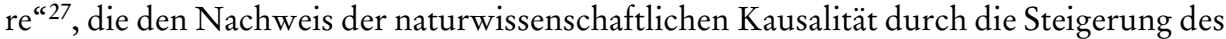
Risikos für das bedrohte Rechtsgut „Leben“ substituiert, hält die Rechtsprechung, die der Risikoerhöhungslehre bekanntlich schon grundsätzlich nicht folgt ${ }^{28}$, zu Recht für nicht zulässig.

Demnach scheidet in den genannten Fällen eine Strafbarkeit wegen eines vollendeten Tötungsdeliktes mangels Kausalität aus ${ }^{29}$. Dies gilt auch dann, wenn man den vorliegenden Sachverhalt nach den für die Unterbrechung rettender Kausalverläufe entwickelten Regeln beurteilt ${ }^{30}$. Nach diesem „heikelsten Fall für die Äquivalenztheorie“31 ist der Kausalzusammenhang zwischen dem Eingriff in den rettenden Kausalverlauf und dem tatbestandsmäßigen Erfolg lediglich unter der engen Voraussetzung zu bejahen, dass der vom Täter unterbundene Kausalverlauf den tatbestandsmäßigen Erfolg mit an Sicherheit grenzender Wahrscheinlichkeit verhindert hätte ${ }^{32}$. Wie dargelegt, fehlt es an dieser Sicherheit in den vorliegenden Fallgestaltungen.

Vor diesem Hintergrund bleibt zu überlegen, ob eine Strafbarkeit der die Manipulationen vornehmenden Mediziner wegen eines versuchten Tötungsdelikts in Betracht kommt.

23 BGH NStZ 1985, 26, 27.

24 Gegenwärtig werden Ein-Jahres-Überlebensraten von 80-90\%, 5-Jahres-Überlebensraten von

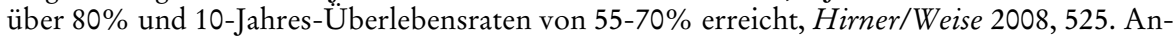
dererseits liegt die Sterberate in den ersten 30 Tagen unmittelbar nach erfolgter Lebertransplantation nach Aussagen der Patientenplattform am Transplantations-Zentrum Heidelberg bei ca. $\%$, http://www.klinikum.uni-heidelberg.de/FAQs.4197.0.html.

25 So kann es durch die Lebertransplantation von einer vorbestehenden Organdysfunktion zu einem vollständigen Organausfall oder zu Infektionen kommen. Der postoperative Verlauf ist durch die notwendige Immunsuppression mit einer Schwächung des Immunsystems verbunden, näher: Huber 2010, 4.

26 Das OLG Braunschweig (Az 9 Ws 49/13) weist darauf hin, dass Patienten mit dem denkbar schlechtesten MELD-Score von 40 Punkten mit 98\% Wahrscheinlichkeit in den nächsten drei Monaten versterben.

27 Befürworter: Brammsen, MDR 1989, 123; Otto, $\mathbb{\$}$ 9, Rn. 100ff.; Stratenwerth 1973, 227.

28 BGHSt 11, 1-7; BGHSt 24, 31-37.

29 Ebenso Kudlich NJW 2013, 918.

30 Roxin 2006, $\mathbb{} 11$ Rn. 35.

31 Roxin 2006, \$11 Rn. 35.

32 Roxin 2006, \$11 Rn. 35; so auch zur ausnahmsweisen Zulässigkeit des Hinzudenkens von hypothetischen Ersatzbedingungen $K \ddot{u} h l$ in Lackner/Kühl 2011, Vorbem. zu $\$ 13$ ff., Rn. 10. 


\section{Versuchter Totschlag}

\section{Die Argumente des Oberlandesgerichts Braunschweig}

Das Oberlandesgericht Braunschweig ${ }^{33}$ bejaht im Rahmen seiner Prüfung des Vorliegens eines dringenden Tatverdachts einen bedingten Tötungsvorsatz und leitet hieraus die Schlussfolgerung ab, vorsätzliche Falschangaben gegenüber der gem. $\$ 12$ Transplantationsgesetz zuständigen Vermittlungsstelle „könnten als versuchte Tötung zum Nachteil dadurch übergangener Patienten bewertet werden“.

Der die Datenmanipulation vornehmende Mediziner habe es für möglich gehalten, dass „andere Menschen sterben“ “. ${ }^{34}$ Gerade ihm als ausgewiesenen Spezialisten für Lebertransplantationen sei bekannt gewesen, dass ,jeder Tag, jede Stunde, sogar jede Minute ... lebenswichtig“ sei und „jede Verzögerung, sei sie auch noch so gering“, den auf die Organspende wartenden Patienten „dem Tod unmittelbar näher“ bringe ${ }^{35}$. Auch das voluntative Vorsatzelement liege vor. Es sei „dringend wahrscheinlich, dass der Beschuldigte in jedem einzelnen Fall billigend in Kauf genommen hat, dass jeweils eine oder mehrere Personen, die aufgrund ihrer Erkrankung sonst bevorzugt ein Organ hätten bekommen sollen, ein Organ nicht mehr rechtzeitig erhalten und infolgedessen versterben“36.

\section{Kritik der „Versuchslösung“}

Der Weg über die „Versuchslösung“ stellt sich nach hier vertretenem Standpunkt als Umgehungsstrategie zur Kompensation der Kausalitätsproblematik dar und ist abzulehnen.

Das Oberlandesgericht begrenzt die Vorsatzprüfung auf eine Analyse des Wissens und Wollens im Hinblick auf den tatbestandsmäßigen Erfolg des $\$ 212$ StGB. Schon dies ist, wie jüngst $S c h r o t b^{37}$ hervorgehoben hat, insbesondere vor dem Hintergrund der „Hemmschwellentheorie“ ${ }^{38}$ fraglich, denn Ärzte wollen primär Leben retten, nicht Leben zerstören. Weiterhin liegt der Argumentation des Oberlandesgerichts der Sache nach die so nicht tragfähige Formel zugrunde, nach der bei Fehlen der Kausalität oder objektiven Zurechnung und gleichzeitig gegebenem Erfolgsvorsatz ohne weiteres Versuch vorliegt. Dass diese Annahme nicht zwingend ist ${ }^{39}$, zeigt bereits der Blick auf die Fallgestaltungen der eigenverantwortlichen Selbstschädigung. Ist der Zurechnungszusammenhang zwischen der Gefahrschaffung durch den Täter und dem Erfolgseintritt durch eigenverantwortliches Handeln, z.B. des Geschädigten, unterbrochen, wird die Kom-

33 OLG Braunschweig, Beschluss vom 20.03.2013 - Ws 49/13 (juris Rn. 34).

34 OLG Braunschweig, Beschluss vom 20.03.2013 - Ws 49/13 (juris Rn. 39).

35 OLG Braunschweig, Beschluss vom 20.03.2013 - Ws 49/13 (juris Rn. 40).

36 OLG Braunschweig, Beschluss vom 20.03.2013 - Ws 49/13 (juris Rn. 44).

37 Schroth NStZ 2013, 437ff.

38 BGH NStZ 1983, 365ff.; NStZ 1983, 407ff.; NStZ 2006, 444; NStZ 2009, $264 f f$.

39 Näher Kübl 2005, \$15, Rn. 10.

NK 25. Jg. 4/2013 
pensation des möglicherweise unbilligen Ergebnisses (Straflosigkeit) durch Rückgriff auf die Versuchsstrafbarkeit zu Recht nicht in Betracht gezogen ${ }^{40}$.

Die auch im Organtransplantationsskandal einschlägige Begründung für dieses Ergebnis liegt im fehlenden Vorsatz im Hinblick auf den vom Täter beherrschbaren Kausalverlauf. Sie beruht mithin auf einer Prüfung, die vom Oberlandesgericht Braunschweig schlicht übersprungen wurde. Denn auch beim Versuch muss der Täter sich Tatumstände vorstellen, bei deren Verwirklichung der volle Unrechtstatbestand des bestimmten Verbrechens erfüllt wäre. ${ }^{41}$ Hierzu gehört auch der Kausalverlauf in seinen wesentlichen Zügen. Ist der Kausalverlauf aufgrund der Vielschichtigkeit des denkbaren Ausgangs eines vom Täter in Gang gesetzten Geschehens aber bereits ex post nicht nachweisbar, kann dieser für den Täter ex ante nicht in seinen wesentlichen Zügen voraussehbar sein. Die Kausalverlaufsprognose aus Tätersicht kann nicht weiter gehen als der kausale Regress aus der Perspektive des Richters. Der manipulierende Arzt wird daher allenfalls für möglich halten und billigen, dass ein Dritter aufgrund seiner Manipulation kein Spenderorgan erhält ${ }^{42}$. Er wird aber nicht den Kausalverlauf voraussehen können, dass deshalb auch ein Mensch zu Tode kommt, der durch die Organtransplantation überlebt hätte. Denn wie oben bereits ausgeführt, kommen insoweit zu viele Verlaufsvarianten ${ }^{43}$ mit glücklichem oder unglücklichem Gesamtausgang des Geschehens im Hinblick auf das von $\$ 212$ StGB geschützte Rechtsgut in Betracht.

Vor diesem Hintergrund wurde gegen den Verantwortlichen im Göttinger Verfahren zu Unrecht Untersuchungshaft wegen des Versuchs des Totschlags verhängt. Auch weitere Straftatbestände sind nach dem oben genannten Sachverhalt nicht ersichtlich. Mangels Kausalität kommt der Rückgriff auf den Tatbestand der Körperverletzung mit Todesfolge, $\$ 227 \mathrm{StGB}$, oder auf Körperverletzung gem. $\$ 223$ StGB nicht in Betracht, aus demselben Grund scheiden auch fahrlässige Tötung oder fahrlässige Körperverletzung aus. Auch Urkundenstraftaten sind nicht einschlägig. Fehlerhaft eingetragene Daten sind schriftliche Lügen und fallen demnach nicht unter $\$ \$ 267 \mathrm{ff}$. StGB ${ }^{44}$, mangels „Gebrauch bei einer Behörde“ ist auch $\$ 278$ StGB in der Fassung de lege lata nicht einschlägig ${ }^{45}$. Ob strafbarer Organhandel gem. $\$ 17$ Transplantationsgesetz vorliegt, ist Tatfrage und noch nicht abschließend geklärt ${ }^{46}$.

40 BGHSt 32, 262-267.

41 A.A. z.B. Frisch 1983, 97f.; kritisch gegen die Vorsatzkonzeption Frischs Schneider 2004, $177 \mathrm{ff}$.

42 Schon diese Annahme ist indessen fraglich, denn aufgrund des beschleunigten Allokationsverfahrens, das nach Angaben Schroths (NStZ 2013, 439) im Jahr 2012 in 40\% der Organvergaben zum Einsatz kam, ist häufig nicht klar, ob evtl. ein Patient mit einem entfernteren Listenplatz nicht doch berücksichtigt werden kann.

43 Zu Recht wird auch von Kudlich, NJW 2013, 917, 918 auf die „Unüberblickbarkeit bzw. Unbeherrschbarkeit (der) weitreichenden Kausalketten“ verwiesen.

44 Kudlich NJW 2013, 919.

45 Schroth NStZ 2013, 437, 447.

46 Schroth NStZ 2013, 437,444 ff. 


\section{Rechtspolitische und gesundheitsökonomische Schlussfolgerungen}

Der Organspendeskandal lässt sich nach alledem mit dem Strafrecht nach den zur Tatzeit geltenden Bestimmungen nicht bewältigen. Vor diesem Hintergrund hat der Gesetzgeber jüngst eine Verschärfung des Transplantationsgesetzes vorgenommen, indem er mit $\$ 19$ Abs. 2a TPG ${ }^{47}$ einen Straftatbestand der Manipulation von Patientendaten geschaffen hat ${ }^{48}$. Danach wird mit Freiheitsstrafe bis zu zwei Jahren oder mit Geldstrafe bestraft, wer absichtlich entgegen $\ 10$ Abs. 3 Satz 2 TPG den Gesundheitszustand eines Patienten erhebt, dokumentiert oder übermittelt. Erfasst werden demnach die unrichtige Erhebung und Dokumentation des Gesundheitszustands sowie die Übermittlung eines unrichtigen Gesundheitszustands. Ein solcher Tatbestand, der den Verstoß von Allokationsrichtlinien ahndet, existiert in der Schweiz bereits seit dem Jahr 2004 ${ }^{49}$. Im strafrechtlichen Schrifttum wird ferner eine Erweiterung des Anwendungsbereichs des $\$ 278$ StGB (Ausstellen unrichtiger Gesundheitszeugnisse) erwogen ${ }^{50}$.

Allerdings erscheint es darüber hinaus angebracht, generelle Bedenken an der Verfassungsmäßigkeit ${ }^{1}$ und medizinischen Praktikabilität ${ }^{52}$ des gegenwärtigen Allokationssystems zu klären. Offensichtlich ist das bisherige System geeignet, bei den wenigen Spezialisten, die in Deutschland die Organtransplantation beherrschen, Neutralisierungsstrategien Vorschub zu leisten. So äußert sich der Beschuldigte im Göttinger Verfahren, viele Transplantationsmediziner seien darüber „frustriert“, dass Patienten mit „echtem MELD Score 40“ (Lebererkrankung im Endstadium, höchster rechnerischer Wert) „mit großer Regelmäßigkeit das transplantierte Organ mit ins Grab nehmen“. Daher sei „eine Organzuteilung bei einem MELD-Wert von schon 30 - 35 wegen der dann wesentlich besseren Überlebenswahrscheinlichkeit medizinisch sinnvoller “53. Vor

47 \$19 TPG in der Fassung vom 15.07.2013.

48 http://www.bundestag.de/presse/hib/2013_06/2013_326/02.html, abgerufen am 25.09.2013.

49 Art. 69 S. 1 Nr. g) des Schweizer Transplantationsgesetzes.

50 Schroth, NStZ 2013, 437, 447.

51 Vgl. Schroth, NStZ 2013, 437, 440, der Kritik an der Unbestimmtheit des $\$ 12$ Abs. 3 TPG übt. Die Vorschrift sieht vor, dass die vermittlungspflichtigen Organe von der Vermittlungsstelle nach Regeln, die dem Stand der Erkenntnisse der medizinischen Wissenschaft entsprechen, insbesondere nach Erfolgsaussicht und Dringlichkeit für geeignete Patienten zu vermitteln sind. Insofern hebt Schroth insbesondere die Widersprüchlichkeit der Allokationskriterien „Dringlichkeit“ und „Erfolgsaussicht“ hervor. Zudem bestünden erhebliche Zweifel an der zutreffenden Wahrnehmung der parlamentarischen Verantwortung, indem in \16 TPG die Kompetenzen zur Aufstellung von Regeln zur Aufnahme in die Warteliste und zur Organvermittlung an die Bundesärztekammer übertragen worden sind; ebenso: http://www.taz.de/! 122022, abgerufen am 24.09.2013.

52 Zur Orientierung der Vergabe knapper Spenderorgane an dem Kriterium Dringlichkeit oder Erfolgsaussicht: Dannecker/Streng JZ 2012, 444 ff.; Kritik der Mediziner an Dringlichkeitskriterium: http://www.faz.net/aktuell/gesellschaft/kriminalitaet/transplantationsskandalwir-verlieren-patienten-12557026.html, abgerufen am 25.09.2013.

53 OLG Braunschweig, Beschluss vom 20.03.2013 - Ws 49/13 (juris Rn. 18). Missstände werden auch von der Deutschen Transplantationsgesellschaft e.V. aufgezeigt, vgl. bereits den offenen Brief an Mitglieder des Deutschen Bundestages vom 13.10.2011.

NK 25. Jg. 4/2013 
diesem Hintergrund wird offensichtlich die stille Revolte durch „Frisieren“ der Patientendaten von einigen Betroffenen als einzige Lösung ihres Dilemmas angesehen.

Die weitergehende Verrechtlichung der Transplantationsmedizin ist aber weder der einzige noch der erfolgversprechendste Weg, um derartige Manipulationen zukünftig wirksam zu verhindern. Die Sachverhalte zeigen auf, dass in deutschen Krankenhäusern, insbesondere in Universitätskliniken, noch immer gravierende Defizite im Bereich Compliance vorhanden sind und daher günstige Tatgelegenheiten - auch für derartige Manipulationen - entstehen. Eine einschlägige Untersuchung des Verfassers zeigt, dass der Krankenhaussektor im Branchenvergleich das Schlusslicht hinsichtlich der Prävalenz von Compliance in deutschen Unternehmen bildet. Insbesondere Hinweisgebersysteme, die das einzige bottom-up Instrument und damit Augen und Ohren der Unternehmensspitze darstellen, sind deutlich unterrepräsentiert ${ }^{54}$. Nur rd. 26\% der Krankenhäuser verfügen (abgesehen von sog. Critical Incident Reporting Systems, die für die Meldung sicherheitsrelevanter Ereignisse im Krankenhaus genutzt werden können) über Hinweisgeberstrukturen oder vordefinierte Berichtswege für Compliance Verstöße. Vor diesem Hintergrund wird der Entstehung günstiger Tatgelegenheiten Vorschub geleistet, weil Entdeckung nicht zu befürchten ist. Hinzu kommt, dass sich in den meisten Krankenhäusern und insbesondere im Bereich der Universitätsmedizin noch immer ein autokratischer Führungsstil der Ordinarien, Chef- und Oberärzte hält, der ein wirksames „bottom-up Kontrollvakuum“ erzeugen kann ${ }^{55}$. Symptomatisch hierfür ist wiederum der Göttinger Fall. Dort hatten Mitarbeiter auf niedrigeren Hierarchiestufen Bedenken und einen Manipulationsverdacht betreffend an Eurotransplant zu meldender Daten gegenüber ihren Vorgesetzten angesprochen und waren mit den Worten beschwichtigt worden, sie sollten „entspannt bleiben“, man wolle schließlich „nur Menschen helfen“. „Dies ist eine ärztliche Anordnung, die sie zu befolgen haben!“56

Die Implementierung von Compliance Maßnahmen in Krankenhäusern steht aber vor hohen Hürden. In Universitätskliniken ist schon die Frage der Zuständigkeit nicht leicht zu klären, sodass entsprechende Anstrengungen bisweilen an Interessenkonflikten zwischen Ordinarien, dem Dekanat der Medizinischen Fakultät, dem Rektorat der Universität und der Verwaltung des Universitätsklinikums scheitern. Zudem bestehen vielfach Spannungen zwischen den Leistungserbringern (Ärzten) und den Leistungsverwaltern (kaufmännische Leitung des Krankenhauses), sodass Initiativen der Unternehmensleitung zur Etablierung von Compliance Strukturen auf Widerstand der Ärzte stoßen, die derartige Maßnahmen als Bevormundung und auch als Beschneidung wissenschaftlicher und ökonomischer Freiheiten empfinden.

Aus diesem Grund dürfte insbesondere im Zusammenhang mit den Manipulationen bei der Organvergabe eine Initiative zu mehr Compliance mit den gesetzlichen Vorgaben nur im Fall einer Reform von innen erfolgversprechend sein. Entsprechende Vorstöße gehen gegenwärtig zum Beispiel von der Deutschen Transplantationsgesellschaft e.V.

54 Näher: Schneider/Grau/Kißling CCZ 2013, 48 ff.

$55 \mathrm{Zu}$ diesen Mechanismen eingehend: Schneider/John 2013.

56 OLG Braunschweig, Beschluss vom 20.03.2013 - Ws 49/13 (juris Rn. 26).

THEMEN 
aus. $\mathrm{Zu}$ erwähnen sind insofern die Anerkennung freiwilliger unangekündigter Kontrollen insbesondere der Lebertransplantationszentren und Initiativen zur Einführung des Mehraugenprinzips.

Auch vor diesem Hintergrund ist es daher angezeigt, auf Alternativen zum Strafrecht zu setzen. Schließlich gibt es keinen empirischen Hinweis darauf, dass eine Prävention mit dem Mitteln des Strafrechts im Bereich der Medizin und Wirtschaft erfolgversprechender ist als im Spektrum der Elends- und Straßenkriminalität.

\section{Literatur}

Brammsen Erfolgszurechnung bei unterlassener Gefahrverminderung durch einen Garanten, in: MDR 1989, $123 \mathrm{ff}$.

Dannecker/Streng Rechtliche Möglichkeiten und Grenzen einer an den Erfolgsaussichten der Transplantation orientierten Organallokation, in: JZ 2012, 444ff.

Frisch (1983) Vorsatz und Risiko, Grundfragen des tatbestandsmäßigen Verhaltens und des Vorsatzes : zugleich ein Beitrag zur Behandlung außertatbestandlicher Möglichkeitsvorstellungen.

Hirner/Weise (2008) Chirurgie, 2. Aufl.

Huber (2010) Risikoanalyse für schwere perioperative Komplikationen nach Lebertransplantation anhand des Patientengutes der Universitätsklinik Tübingen, Dissertation Joecks/Miebach (Hrsg.) (2012) Münchener Kommentar zum StGB, Band 4, 2. Aufl.

Kaufmann (1959) Die Dogmatik der Unterlassungsdelikte

Kindhäuser (2011) Strafrecht Allgemeiner Teil, 5. Aufl.

Kudlich Die strafrechtliche Aufarbeitung des Organspendeskandals, in: NJW 2013, $217 \mathrm{ff}$.

Kühl (2005) Strafrecht Allgemeiner Teil, 5. Aufl.

Lackner/Kühl (Hrsg.) (2011) Strafgesetzbuch Kommentar, 27. Aufl.

Laufbütte/Rissing-van Saan/Tiedemann (Hrsg.) (2007) Leipziger Kommentar zum StGB, Band I, 12. Aufl.

Otto (2004) Grundkurs Strafrecht. Allgemeine Strafrechtslehre, 7. Aufl.

Roxin (2006) Strafrecht Allgemeiner Teil, Band I, 4. Aufl.

Schneider (2004) Kann die Einübung in Normanerkennung die Strafrechtsdogmatik leiten? 
Schneider/Grau/Kißling „Der Schock von Berlin saß tief!“ Ergebnisse eines empirischen Forschungsvorhabens zu Compliance im Gesundheitswesen und in der Pharmaindustrie, in: CCZ 2013, $48 \mathrm{ff}$.

Schneider/John (2013) Das Unternehmen als Opfer von Wirtschaftskriminalität. Eine viktimologische Untersuchung: Public und Private Sector im Vergleich

Schönke/Schröder (Hrsg.) (2010) Strafgesetzbuch Kommentar, 28. Aufl.

Schroth Die strafrechtliche Beurteilung der Manipulationen bei der Leberallokation, in: NStZ 2013, $437 \mathrm{ff}$.

Spickhoff (Hrsg.) (2011) Beck'scher Kurz-Kommentar Medizinrecht

Stratenwerth (1973) Bemerkung zum Prinzip der Risikoerhöhung, in: Lackner u. a. (Hrsg.), Festschrift für Gallas, 227ff.

Wessels/Beulke (2012) Strafrecht Allgemeiner Teil, 42. Aufl.

Wessels/Hettinger (2011), Strafrecht Besonderer Teil 1, 35. Aufl.

Kontakt:

Prof. Dr. Hendrik Schneider

Wiss. Mit. Josephine Busch

Lebrstubl für Strafrecht, Strafprozessrecht, Kriminologie, Jugendstrafrecht und Strafvollzugsrecht

Universität Leipzig

Juristenfakultät

Burgstraße 27

04109 Leipzig 